\title{
Analogy between non-alcoholic steatohepatitis (NASH) and hypertension: a stepwise patient-tailored approach for NASH treatment
}

Yaron Ilan

Hadassah Hebrew University Medical Center, Jerusalem, Israel

\begin{abstract}
Non-alcoholic steatohepatitis (NASH) is a common liver disorder worldwide. Although there has been improvement in our understanding of the natural history and pathogenesis of the disease, there is still no approved therapy for NASH. NASH shares many similarities with primary hypertension, in that both are extremely common disorders that can easily lead to serious complications if left untreated. Both conditions are viewed as "silent killers", because the disease can progress over a period of time prior to the occurrence of potentially deadly outcomes. While attempts to find the "miracle pill" for NASH are unrealistic, we can make an analogy with the "stepwise combination" approach developed over the last few decades for the treatment of hypertension. In the present review, we summarize some of the similarities in the concepts that underlie NASH and hypertension. The development of a stepwise patient-tailored method for the treatment of NASH is presented.
\end{abstract}

Keywords Non-alcoholic steatohepatitis, hypertension, treatment, non-alcoholic fatty liver disease

Ann Gastroenterol 2018; 31 (3): 1-9

\section{Introduction}

Non-alcoholic fatty liver disease (NAFLD) is defined as the presence of a significant amount of lipid accumulation in the liver without significant alcohol consumption [1]. NAFLD represents two distinct entities with two different prognoses: the first is simple fat accumulation in the liver; and the second is non-alcoholic steatohepatitis (NASH), which comprises necro-inflammation and may lead to fibrosis, cirrhosis and hepatocellular carcinoma (HCC) $[1,2]$. NAFLD is the most common cause of chronic liver disease worldwide [3]. Despite progress in the understanding of the pathophysiology of the disease and diagnostic methods, there is still no approved therapy $[4,5]$.

Gastroenterology and Liver Units, Department of Medicine, Hadassah Hebrew University Medical Center, Jerusalem, Israel

Conflict of Interest: This work was supported in part by The RomanEpstein Liver Research Foundation. Dr Yaron Ilan is the Medical Director of Exalenz and a consultant for Immuron, Teva, Enzo Biochem, Protalix, Therapix, Nasvax, Medial, Tiziana, JTI, and Natural Shield

Correspondence to: Yaron Ilan, MD, Gastroenterology and Liver Units, Department of Medicine, Hebrew University-Hadassah Medical Center, Ein-Karem, POB 1200 Jerusalem, Israel, e-mail: ilan@hadassah.org.il

Receied 2 August 2017; accepted 24 January 2018;

published online 15 March 2018

DOI: https://doi.org/10.20524/aog.2018.0248
Hypertension (HTN) has been recognized for much longer than NASH and the two conditions share many similarities. We describe some of the common features of both diseases in an effort to determine what hepatologists can learn from HTN experts with regard to treatment strategy.

\section{NAFLD and HTN are two global epidemics}

NAFLD and HTN are highly common disorders. Approximately $90 \%$ of HTN cases are classified as essential HTN, where the precise cause is unknown [6]. Both secondary liver steatosis due to non-NAFLD causes (e.g., HCV genotype 3, Wilson's, etc.) and secondary HTN are less common. NAFLD is one of the main causes of chronic liver disease globally [7]. A recent study determined that the prevalence of NAFLD was $24 \%$ and that the global prevalence positively correlated with the gross national income per capita: Europe observed a higher prevalence (28\%) than the Middle East (12\%) and East Asia (19\%) [8]. Like NAFLD, HTN is a major contributor to the global burden of disease and mortality [9]. A national survey estimates the prevalence to be $16-36 \%$ [10]. A recent review found that the prevalence of treatment-resistant HTN $(\mathrm{RH})$ is between $13 \%$ and $16 \%$ [11]. NAFLD and HTN are strongly associated with obesity and insulin resistance states including diabetes. HTN is one of the criteria for metabolic syndrome [12]. NAFLD is associated with components of 
metabolic syndrome. Sixty-six percent of patients older than 50 years with diabetes or obesity are thought to have NASH with advanced fibrosis [2]. However, arterial HTN, which is among the various components of metabolic syndrome, was found to be the least closely associated with NAFLD [13]. More advanced stages of NAFLD are associated with HTN $[14,15]$. Angiotensinogen gene haplotype is associated with the prevalence of Japanese NASH [16]. In the absence of major risk factors for liver disease, NAFLD is a frequent finding in primary aldosteronism. These patients are more insulin resistant and have a higher prevalence of NAFLD [17].

\section{NAFLD and HTN are associated with a high risk of cardiovascular and kidney diseases}

NAFLD and HTN are associated with similar target organ damage. The clinical burden of NAFLD is the result of liverrelated morbidity or mortality, although the majority of deaths in NAFLD patients are related to cardiovascular disease (CVD) and cancer [18]. NAFLD is a risk factor for extrahepatic diseases such as CVD, chronic kidney disease (CKD), colorectal cancer, and endocrinopathies, which include type 2 diabetes mellitus (T2DM), thyroid dysfunction, colorectal neoplasms, and osteoporosis $[18,19]$. The prevalence of NAFLD is three times higher in patients with T2DM [20]. Patients with NAFLD are also at a higher risk for atherosclerosis [21]. It has been suggested that patients with NAFLD should undergo periodic CVD risk assessment [22]. Similarly, the association between systolic and diastolic HTN and the risk of CVD and renal disease is well known. HTN is associated with a higher risk of acute kidney injury [23]. The prevalence of HTN is higher and its control is more difficult with poor kidney function. The presence and severity of CKD increases treatment resistance [24]. Obese individuals are more likely to be at increased risk for developing NAFLD, HTN, CVD, and CKD [25].

A recent Consensus analyzed the effect of treatment using statins alone, or in combination with pioglitazone and other drugs, on CVD as a main cause of death in patients with NAFLD, and on liver-related complications of NAFLD or $\mathrm{NASH}$, including cirrhosis and hepatocellular carcinoma [26]. This Consensus suggested a tailored "HTN-like" therapy.

Prevalent NAFLD may be seen early in the development of HTN, even in the absence of other metabolic risk factors. Controlling blood pressure among non-obese hypertensive patients may be beneficial in preventing or limiting NAFLD [27]. The prevalence of NAFLD among persons with normal blood pressure, prehypertension (PHT), and HTN was $16.5,37.5$, and $59.3 \%$, respectively. In multivariate analyses, PHT and HTN were associated with elevated odds of NAFLD.

Data support the paradigm of NAFLD as a strong determinant for the development of the metabolic syndrome, which has potentially relevant clinical implications for diagnosing, preventing and treating metabolic syndrome. Longitudinal studies support the association of NAFLD with either T2DM or metabolic syndrome, and suggest that NAFLD precedes the development of both conditions [28].
Based on data from 118 consecutive biopsy-proven NAFLD patients, the metabolic syndrome, the homeostatic model assessment of insulin resistance, serum total cholesterol, and serum uric acid were identified as independent predictors of $\mathrm{NASH}$ and its individual histological lesions, including fibrosis. These factors were suggested as pathogenic drivers of NASH and as potential targets for treatment [29].

\section{NAFLD and HTN are multifactorial diseases}

The pathogenesis of NAFLD and NASH and the mechanisms that lead to liver injury, fibrosis, and HCC are the result of a complex interplay between host and environmental factors. Genetic, biochemical, immunological, and molecular events are associated with disease progression [30]. The pathogenesis of HTN, similar to that of NAFLD, involves genetic, endocrine, metabolic, immune and nervous system parameters.

In NAFLD, environmental factors play a major role in genetically susceptible populations. Diet plays a role in this process, as fructose exacerbates NAFLD, while the Mediterranean diet exerts a protective effect [31]. A diet rich in simple carbohydrates, saturated fat, and highly processed food on a background of several genetic variants presents a risk for NAFLD [32]. De novo lipogenesis, i.e., hepatic triglyceride synthesis, accounts for only $25 \%$ of hepatic triglycerides, while the role of lipolysis as a factor contributing to hepatic triglyceride storage remains unconsidered [33]. Increased liver fat induces mitochondrial metabolism and glyceroneogenesis and its conversion from lactate to glycerol is used as a substrate for gluconeogenesis. Hepatic fat removal, i.e., increased mitochondrial $\beta$-oxidation and autophagy, contribute to liver damage [34-38]. NAFLD typically results from fatty changes observed in the absence of competing steatogenic factors in dysmetabolic individuals [39]. Reduced metabolic adaptability has been described, in which liver fat accumulation increases the demands on the liver to control metabolic responses [40].

For HTN, a defect in sodium excretion by the kidney is central to the pathogenesis. A congenital reduction in nephron number, obesity, hyperleptinemia, a diet rich in salt and fructose, increased sympathetic nervous system tone, hyperuricemia, renal arteriolar vasoconstriction, and intrarenal activation of the renin-angiotensin system are significant in the pathogenesis.

Gene expression profiling and genome-wide association studies have identified disease pathways and polymorphisms in genes that determine NAFLD progression [41,42]. The polygenic background with multiple independent modifiers determines disease prognosis. The risk allele frequencies of NAFLD-associated single nucleotide polymorphisms were analyzed in distinct populations that have high risk scores [43]. Patatin-like phospholipase domain-containing 3 (PNPLA3) has both anabolic and catabolic activities in lipid metabolism and has been reported to be linked with liver fat content [44]. A link from the 148 isoleucine to a methionine protein variant of PNPLA3, NAFLD and fibrosis has been described. Genes involved in lipolysis, adipokine, and cytokine production 
are being explored [45-47]. Target genes for peroxisome proliferator-activated receptor (PPAR) $\alpha$, a ligand-activated transcription factor, are involved in fatty acid metabolism [48]. During PPAR $\alpha$ activation, the combination with PPAR $\beta / \delta$ agonism improves steatosis, inflammation and fibrosis in NAFLD [49].

Genetic congenital (fetal programming) and acquired mechanisms for defects in natriuresis increase the risk for development of HTN. Genetic polymorphisms regulate sodium excretion. It is estimated that $30 \%$ of the variance in blood pressure relates to genetic factors [9]. Genome-wide studies identified more than 65 loci affecting blood pressure [50].

Chronic low-level inflammation is associated with the metabolic syndrome [51]. While the liver provides a "tolerogenic" environment, abnormal activation of innate immune cells triggers inflammation that contributes to hepatic injury, fibrosis, and carcinogenesis [52,53]. Innate immune cells, the adaptive system, liver macrophage Kupffer cells, stellate cells, and dendritic cells contribute to the development of fibrosis. Adiponectin, leptin and ghrelin, resistin, visfatin and retinol-binding protein 4 , along with tumor necrosis factor (TNF)- $\alpha$, interleukin (IL)-6, IL-1, and IL-18 are some of the major factors involved [53].

Like NAFLD, HTN is linked with inflammation. Hence, both the innate and adaptive immune responses participate in this process [54]. Oxidative stress and endothelial dysfunction are contributing factors for the development of HTN [6]. Increased sympathetic and/or decreased parasympathetic outflow or low-grade infections generate neoantigens and damage-activated or pathogen-activated molecular patterns, which trigger Toll-like receptors on innate cells [54]. Innate responses, mediated by monocytes, macrophages, dendritic cells, and natural killer cells, contribute to inflammation by activating a T-cell-mediated adaptive immune response [54]. Activation of the sympathetic nervous system, aging and elevated aldosterone are potentially proinflammatory. Intrarenal T cells are associated with persistence of HTN, suggesting induction of a local autoimmune response to neoantigens, such as heat shock protein 70 and protein aggregates resulting from lipid peroxidation [55].

NAFLD has also been linked with alterations in the nervous system. Altered neuroendocrine and autonomic signals controlled by the suprachiasmatic nucleus contribute to steatosis, obesity and glucose intolerance. Excess free fatty acids in hepatocyte storage lead to lipotoxicity, hepatocyte damage, and apoptosis. Glucagon-like peptide (GLP)-1 analogues stimulate pancreatic $\beta$-cell insulin output and affect the liver's free fatty acid metabolism [56].

Likewise, for HTN, the renal sensory afferent nerves and efferent sympathetic nerves control the extracellular fluid volume and hence the level at which blood pressure is set. Afferent and efferent renal innervation contribute to neural dysregulation of the kidney in RH [57]. Sympathetic neural regulation of renin release and fluid reabsorption in the kidney affect HTN. RH is attributed to aldosterone excess in over $20 \%$ of patients. The function of amiloride-sensitive sodium channels and mineralocorticoid receptors in the systemic vasculature supports aldosterone-mediated $\mathrm{RH}$ [58].
The gut microbiome plays a role in the pathogenesis of both NAFLD and HTN [59]. Altered intestinal permeability supports a link between gut lumen antigenic/toxic substances and systemic and liver inflammation in NAFLD [59]. Patients with NASH have increased Gram-negative microbiome and endotoxemia. Microbial metabolites aid in the development of hepatic steatosis and inflammation, NASH and fibrosis [60]. Short-chain fatty acids, the products of microbial fermentation, enhance intestinal absorption by activating GLP-2 signaling [60]. However, many NASH patients show normal serum endotoxin levels, indicating that endotoxemia may not necessarily be required for the development of NASH [60].

Dysbiosis in the gut microbiota has also been described in association with HTN in both animal models and humans [61]. An abundance of the Firmicutes and Bacteroidetes gut microbes has been found in HTN. Decreases in gut microbiota caused by antibiotics can increase or decrease blood pressure depending on the patient's genotype. Products of the fermentation of nutrients by gut microbiota change the blood pressure by altering the expenditure of energy, intestinal metabolism of catecholamines, gastrointestinal and renal ion transports, and finally, salt sensitivity [61].

\section{NAFLD and HTN are "silent killers" with marked interpatient variability in disease progression}

In NAFLD and HTN, there is considerable interpatient variability in both severity and rate of progression [62]. NAFLD and HTN can be present with different phenotypes as a result of the multiple factors associated with disease progression. Patients with NAFLD can present with simple steatosis, different stages of fibrosis and pre-cirrhotic NASH, compensated cirrhosis, or advanced NASH cirrhosis. The rate of disease progression differs among patients [63]. Likewise, hypertensive patients may have diverse degrees of disease progression and different complications.

For NAFLD and HTN, a substantial proportion of the population is at risk of progressive disease, while the minority experience associated morbidity. In both disorders, the term "silent killer" is used, because in both conditions a relatively long-term "silent disease" may lead to serious complications and increased mortality. NAFLD is associated with a high rate of mortality from CVD, late-stage liver disease and HCC [64]. About one third of patients with early-stage NASH progress to cirrhosis over a period of 5-10 years. Among those who progress to NASH cirrhosis, approximately $25 \%$ develop the major complication of portal HTN within 3 years. HCC is described in the setting of NASH as well as of obesity and diabetes [65]. It has also been suggested that HCC may develop in "silent NAFLD" patients without cirrhosis [66]. The majority of deaths in NAFLD patients are related to CVD and cancer [67]. However, it remains unproven whether NASH carries excess CVD risk compared with simple steatosis [68]. NAFLD is associated with subclinical manifestations of atherosclerosis, including increased intima-media thickness, 
endothelial dysfunction, arterial stiffness, impaired left ventricular function and coronary calcification [69]. HTN is responsible for 7.6 million deaths per year worldwide, $13.5 \%$ of the total, more than any other risk factor [70]. HTN is also responsible for approximately $41 \%$ of CVD-related deaths [61]. HTN is associated with over $50 \%$ of the cases of stroke and coronary heart disease. An association between CVD deaths and relatively low blood pressure was described, which further supports "the silent killer" paradigm of HTN [70].

\section{Difficulties in developing treatments for NAFLD and HTN: from lifestyle modification to a combination drug strategy}

There are challenges to optimizing the treatment strategies for both NAFLD and HTN to improve outcomes and prevent long-term complications. For NASH and HTN, an intervention targeting key environmental factors is required, and both require collaborative efforts from specialists in various medical fields as well as from primary care physicians.

There is no approved therapy for NASH at present [71]. The lack of a valid biomarker for both NAFLD and NASH makes it complex to monitor the effect of therapy on the disease. Hepatologists treating NASH can adopt some of the strategies developed over the last decades for HTN. These treatments are based on three major principles: (i) non-pharmacological management; (ii) a multi-drug combination regimen targeting different disease-relevant pathways; and (iii) a patient-tailored approach to therapy.

i. Guidelines for non-pharmacological management are fundamental in the treatment of HTN. Weight reduction, reduced salt intake, increased dietary intake of fresh fruits and vegetables, increased low-fat dairy intake, physical activity and a reduction in saturated fat and cholesterol intake, along with regular fish intake are recommended for a healthy lifestyle change [7]. Most studies and recommendations suggest an association between salt intake and HTN, in which decreased salt intake has been proven to be effective in decreasing HTN over the years. However, the new "salt controversy" suggests that even "simple" lifestyle modifications are not always straightforward $[9,72,73]$. An important finding was the J-shaped relationship between salt intake, mortality and CVD events. Likewise, lifestyle modifications should be an important part of any therapeutic program in NASH, irrespective of the disease stage [64]. Weight loss can improve the histological changes of NAFLD and can also alleviate NASH [74]. Dietary counseling and regular exercise must be a necessary treatment strategy in all patients.

ii. For HTN, effective implementation of a combination drug regimen, such as triple drug-based therapies, can control blood pressure in about $90 \%$ of patients [9]. HTN patients on multiple drugs have better blood pressure control than patients on monotherapy [75] and also have better protection against CVD. While the recommended combinations of therapies differ, they are all based on variations of any two renin-angiotensin system blockers, calcium-channel blockers, and diuretics. The use of $\beta$-blockers as a major agent is still recommended in Europe. A step-by-step guide on how to manage the increased RH has been developed [7]. These guidelines are based on a large meta-analysis, which determined the effect of therapy on morbidity and mortality. The inferiority of low-dose thiazides was shown when compared with other drug classes and also compared with other diuretics [76]. An additional important factor in drug selection that may also be relevant for patients with NASH is the potential side effects; for example, higher-dose thiazides have fallen out of use because of their detrimental effects on potassium levels. The guidelines of the European Society of Hypertension, the American and International Society of Hypertension, and the Eighth Joint National Committee (JNC8) proposed the use of two drugs in combination to initiate therapy for a large proportion of patients [77]. The use of single-pill combinations of two antihypertensive agents is associated with considerably better adherence [78]. A recent study summarizing 68 randomized controlled HTN trials showed that lowering blood pressure significantly reduced major CVD outcomes independently of the agents used [79]. A significant risk reduction was observed in all stages of HTN. iii. Optimizing drug therapy for HTN needs to be done in a patient-tailored way. The effect of genetic loci and phenotypic features, including ethnicity, on a patient's response to therapy should be considered. The patterns of response to treatment and the rate of complications in patients with HTN differ between White, Hispanic and African American patients [24]. All guidelines make an effort to differentiate the recommendations on the basis of age and ethnic group when they propose the use of combination therapies. Drug selection, however, varies among the different guidelines [80]. Patients with $\mathrm{RH}$ are a subgroup at high risk and a treatment regimen for these patients is being developed [81]. Both drug selection and treatment targets need to be patient-tailored in HTN. Previous guidelines suggested a target of $130 / 80 \mathrm{mmHg}$ or lower for all patients with diabetes or chronic renal failure. Because of insufficient data, the targets were increased to $140 / 85 \mathrm{mmHg}$ and $140 / 90 \mathrm{mmHg}$, respectively [82]. For patients over 60 years of age, the treatment threshold increases to $150 / 90 \mathrm{mmHg}$.

Like HTN, the treatment goals in patients with NAFLD should vary depending on the stage of the disease, the potential risk of progression and any comorbid diseases [64]. Patients with NASH should be targeted for treatment, especially if they have concomitant fibrosis, because they are more likely to develop cirrhosis and HCC than those without fibrosis [64].

Several of the drugs used for the treatment of HTN were shown to exert some beneficial effect in pre-clinical and small proof-of-concept clinical trials. These include losartan [83], pioglitazone in combination with candesartan [84], combined ursodeoxycholic acid and angiotensin-II type 1 receptor blocker [85], angiotensin-receptor blockers [86], 
telmisartan [87], angiotensin II type 1 receptor blocker, and irbesartan [88].

The therapeutic methods being developed for NASH were recently reviewed [89]. Hepatic fat accumulation and metabolic stress are major targets for therapy using peroxisome proliferator-activator receptor agonists (e.g., pioglitazone, elafibranor, saroglitazar). Other drugs target the gut microbiome or the gut immune system [90]. Drugs targeting the bile acid-farnesoid $\mathrm{X}$ receptor axis (obeticholic acid), inhibitors of de novo lipogenesis (aramchol), incretins (liraglutide) and fibroblast growth factor (FGF)-21 and FGF-19 analogs are in various stages of development [89]. Another approach is targeting the inflammation underlying the disease using antioxidants (vitamin E), medications with a target in the TNF- $\alpha$ pathway (emricasan, pentoxifylline) and immune modulators (amlexanox, cenicriviroc, anti-CD3, antiTNF $[90,91]$ ). Antifibrotics (simtuzumab and GR-MD-02) are also being tested with the aim of reversing the fibrosis process. Metformin revealed no biochemical or histological improvement and is not recommended [92]. Bariatric surgery is recommended for morbidly obese patients and leads to a significant improvement in liver histology and metabolic syndrome $[3,93]$.

Several controlled trials have shown some benefits in patients with NASH. The PIVENS trial showed that vitamin E was superior to placebo for the treatment of NASH in adults without diabetes [94]; in the Flint trial, obeticholic acid improved the histological features of NASH [95]; the LEAN trial showed liraglutide to be safe and associated with histological resolution of several parameters of NASH [96]; in the Golden trial, GFT505, a dual agonist of the PPAR $\alpha$ and PPAR $\delta$ isoforms, showed some benefits in subsets of patients [97].

The potential risks of using some of these drugs may prohibit their long-term use in large populations of patients with NAFLD: pioglitazone is associated with weight gain [98]; obeticholic acid may lead to hyperlipidemia in a significant proportion of patients; and vitamin $\mathrm{E}$ is associated with potential long-term risks [4].

\section{Development of a multi-step, patient-tailored drug combination treatment strategy for NASH}

The best treatment for NASH is based on the three principles described above, combining a non-pharmacological approach with patient-tailored multi-drug combination therapy (Fig. 1). The drug combination needs to target inflammatory as well as fibrosis pathways. While resolution of inflammation may also contribute to the inhibition of fibrogenesis, direct antifibrotic drugs are required for reversal of existing liver damage.

Developing the personalized treatment protocols needed in a patient-tailored way is based on genomic, proteomic, metabolomic, lipidomic, and microbiome studies (Fig. 2). Identifying those patients left in the stage of simple steatosis, a benign condition, compared to those at risk for developing liver inflammation, fibrosis, cirrhosis and HCC, is essential [45].

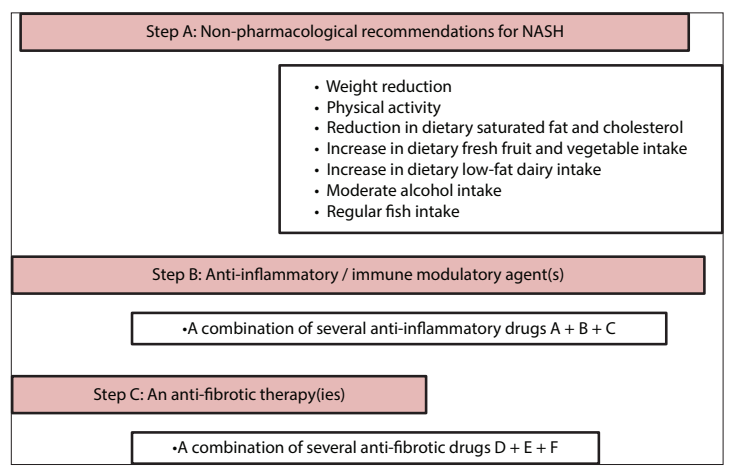

Figure 1 A schematic stepwise approach for the treatment of nonalcoholic steatohepatitis (NASH)

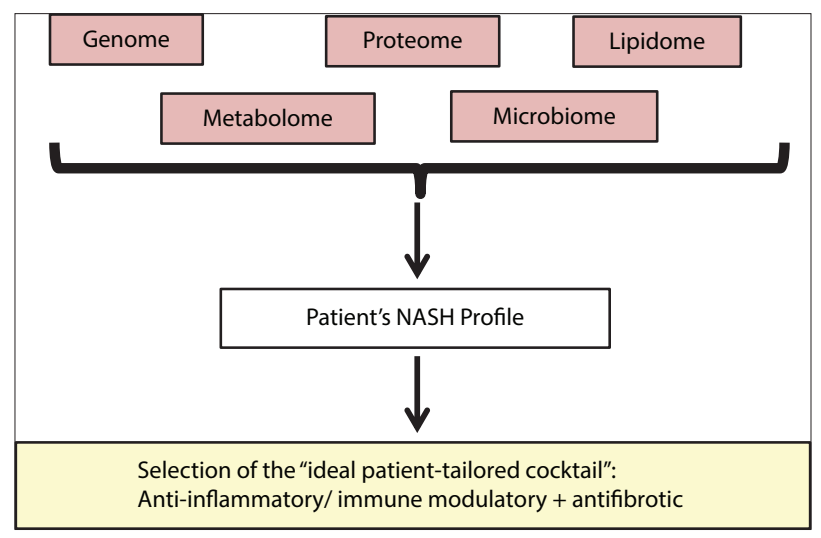

Figure 2 Selection of the ideal anti-inflammatory/immune modulatory and antifibrotic drug combination for patients with nonalcoholic steatohepatitis (NASH) based on a patient-tailored regimen

Analyzing the proteome of the liver mitochondria revealed a platform that distinguished NASH $[99,100]$. Over 550 proteins were identified in the NAFLD proteome, and several were found to be significantly up- and downregulated in NASH [99]. These include apolipoprotein E, insulin-like growth factorbinding protein 3, vitamin D-binding protein and lymphocyte cytosolic protein. These proteins are involved in fatty acid $\beta$-oxidation processes, lipid metabolic processes, cell-cycle arrest, cell polarity maintenance, and adenosine triphosphate/ sex hormone metabolic processes [100]. Liver samples obtained from diabetic and non-diabetic morbidly obese subjects showed a decreased abundance of mitochondrial enzymes, proteins involved in methionine metabolism and a reduction in the oxidative stress response. Patients with T2DM exhibited decreased levels of glutathione, the antioxidant byproduct of methionine metabolism, via the transsulfuration pathway and a higher level of protein and lipid oxidative damage, along with alterations in detoxifying enzymes, carbohydrate metabolism, proteasome subunits and retinoic acid synthesis.

For metabolomics, proteins implicated in transportation, metabolic pathways, acute phase reactions, anti-inflammatory effects, the extracellular matrix, and the immune system are explored [45,101]. Profiling of metabolic products 
using nuclear magnetic resonance spectroscopy and mass spectrometry combined with statistical modeling and topdown systems biology identified metabolic signatures in NAFLD [102]. A small-molecular screen of human liver tissue showed that hydroquinone and nicotinic acid were inversely correlated with histological NAFLD severity [103]. $\gamma$-Glutamyl dipeptides may differentiate between NASH and simple steatosis. A non-targeted metabolomics approach to the plasma from morbidly obese patients undergoing bariatric surgery detected differences between patients with or without NAFLD. Accumulation of lipids in hepatocytes is linked with $\alpha$-ketoglutarate, decreased $\beta$-oxidation energy production, reduced liver function, and altered glucose metabolism [104]. Plasma a-ketoglutarate levels distinguish between those with or without NAFLD. Assessment of the bile acid metabolome indicated a higher total serum bile acid concentration in $\mathrm{NASH}$. Increased taurine- and glycine-conjugated primary and secondary bile acids were identified [105]. The metabolome is also able to identify patients likely to respond to therapy such as vitamin E. At baseline, phenyl-propionic acid and indole-propionic acid levels were directly connected with a subsequent histologic response to vitamin E treatment, while $\gamma$-carboxyethylhydroxychroman levels were inversely related to the response. The end-of-treatment levels of $\gamma$-glutamyl leucine and $\gamma$-glutamyl valine were lower in vitamin $\mathrm{E}$ respondents.

Lipidomic studies characterized the potential relationships between lipotoxicity, inflammation, oxidative stress, and cellular function [106]. Lipidomic data from the portal and systemic blood defined a NASH signature [107]. Increased concentrations of several glycerophosphocholines, glycerophosphoethanolamines, glycerophosphoinositols, glycerophosphoglycerols, lyso-glycerophosphocholines and ceramides were detected in the systemic circulation of NASH subjects. Analysis of lipids from the portal system at the time of surgery revealed limited lipid alterations compared with the systemic circulation, but glycerophosphoethanolamines (PE), and, glycerophosphoglycerols (PG) classes were significantly increased in NASH subjects [107]. Lipid species may also serve as markers of advanced liver disease. An increase in diacylglycerols was demonstrated in NAFLD, supporting their role in the progression of NAFLD and liver fibrosis [108]. Lipid-modifying enzymes used in converting saturated fatty acids to monounsaturated fatty acids are relevant for the development of HCC [109-111]. Increased ratios of long-chain n6-polyunsaturated fatty acids over n3-polyunsaturated fatty acids are a risk factor for both NASH and HCC [112,113].

Dysbiosis and the gut-microbiota-liver network determine the phenotype in patients with NAFLD [114]. The transfer of gut microbiota from lean and obese individuals induced the metabolic features of the donor in the recipients. Bidirectional interactions of the gut microbiota, including with food, bile and the intestinal epithelium, are associated with the progression from steatosis to steatohepatitis, fibrosis, and cancer [115]. Caloric extraction from the diet, intestinal epithelial damage and entry of bacterial components into the portal circulation are important contributors to innate activation, liver inflammation and fibrosis [116]. Gut microbiota-linked compounds, including short-chain fatty acids, bile acids, choline metabolites, indole derivatives, vitamins, polyamines, lipids, neurotransmitters, neuroactive compounds, and hypothalamic-pituitary-adrenal axis hormones, are additional factors [117]. Serum lipid levels of phospholipids, free fatty acids, polyunsaturated fatty acids, especially eicosapentaenoic acid, arachidonic acid, and docosahexaenoic acid, correlate with specific fecal flora. Cytokines, amino acids, vitamins, and fatty acid metabolism also correspond with gut microbiota [117].

The use of a combination of proteome- and/or metabolomeand/or lipidome- and/or microbiome-based data can serve to tailor selected therapies to the appropriate patients in order to increase response rates and avoid exposing patients with low response potential to unnecessary side effects.

\section{Concluding remarks}

Although an effort has been made over the last two decades to understand the natural history and pathogenesis of NAFLD and NASH and to develop noninvasive diagnostic measures, there is still no approved therapy for NASH [4]. Moreover, it appears that the therapies developed to date are unlikely to provide a "one-pill" type of treatment for NASH. The development of patient-tailored "HTN-like" therapy protocols is suggested as a way to offer therapy to patients with different risk levels and this should be our goal for the near future.

\section{References}

1. Masarone M, Federico A, Abenavoli L, Loguercio C, Persico M. Non alcoholic fatty liver: epidemiology and natural history. Rev Recent Clin Trials 2014;9:126-133.

2. Rinella ME. Nonalcoholic fatty liver disease: a systematic review. JAMA 2015;313:2263-2273.

3. European Association for the Study of Obesity (EASO). EASLEASD-EASO Clinical Practice Guidelines for the management of non-alcoholic fatty liver disease. J Hepatol 2016;64:1388-1402.

4. Ratziu V, Goodman Z, Sanyal A. Current efforts and trends in the treatment of NASH. J Hepatol 2015;62:S65-S75.

5. Italian Association for the Study of the Liver (AISF). AISF position paper on nonalcoholic fatty liver disease (NAFLD): Updates and future directions. Dig Liver Dis 2017;49:471-483.

6. Dinh QN, Drummond GR, Sobey CG, Chrissobolis S. Roles of inflammation, oxidative stress, and vascular dysfunction in hypertension. Biomed Res Int 2014;2014:406960.

7. Schneier AT, Citti CC, Dieterich DT. Management and diagnosis of fatty liver disease. Expert Rev Gastroenterol Hepatol 2015;9:671-683.

8. Zhu JZ, Dai YN, Wang YM, Zhou QY, Yu CH, Li YM. Prevalence of nonalcoholic fatty liver disease and economy. Dig Dis Sci 2015;60:3194-3202.

9. Poulter NR, Prabhakaran D, Caulfield M. Hypertension. Lancet 2015;386:801-812.

10. Ibrahim MM, Damasceno A. Hypertension in developing countries. Lancet 2012;380:611-619.

11. Achelrod D, Wenzel U, Frey S. Systematic review and meta-analysis of the prevalence of resistant hypertension in treated hypertensive populations. Am J Hypertens 2015;28:355-361. 
12. Alberti KG, Zimmet P, Shaw J; IDF Epidemiology Task Force Consensus Group. The metabolic syndrome-a new worldwide definition. Lancet 2005;366:1059-1062.

13. Lonardo A, Bellentani S, Argo CK, et al; Non-alcoholic Fatty Liver Disease Study Group. Epidemiological modifiers of nonalcoholic fatty liver disease: Focus on high-risk groups. Dig Liver Dis 2015;47:997-1006.

14. Byrne CD, Targher G. NAFLD: a multisystem disease. J Hepatol 2015;62:S47-S64.

15. Clark JM. The epidemiology of nonalcoholic fatty liver disease in adults. J Clin Gastroenterol 2006;40 Suppl 1:S5-S10.

16. Ono $\mathrm{M}$, Ochi $\mathrm{T}$, Munekage $\mathrm{K}$, et al. Angiotensinogen gene haplotype is associated with the prevalence of Japanese nonalcoholic steatohepatitis. Hepatol Res 2011;41:1223-1229.

17. Fallo F, Dalla Pozza A, Tecchio M, et al. Nonalcoholic fatty liver disease in primary aldosteronism: a pilot study. Am J Hypertens 2010;23:2-5.

18. Armstrong MJ, Adams LA, Canbay A, Syn WK. Extrahepatic complications of nonalcoholic fatty liver disease. Hepatology 2014;59:1174-1197.

19. Musso G, Gambino R, Tabibian JH, et al. Association of nonalcoholic fatty liver disease with chronic kidney disease: a systematic review and meta-analysis. PLoS Med 2014;11:e1001680.

20. Saponaro C, Gaggini M, Gastaldelli A. Nonalcoholic fatty liver disease and type 2 diabetes: common pathophysiologic mechanisms. Curr Diab Rep 2015;15:607.

21. Perazzo H, Poynard T, Dufour JF. The interactions of nonalcoholic fatty liver disease and cardiovascular diseases. Clin Liver Dis 2014;18:233-248.

22. Liu H, Lu HY. Nonalcoholic fatty liver disease and cardiovascular disease. World J Gastroenterol 2014;20:8407-8415.

23. James MT, Grams ME, Woodward M, et al; CKD Prognosis Consortium. A meta-analysis of the association of estimated GFR, albuminuria, diabetes mellitus, and hypertension with acute kidney injury. Am J Kidney Dis 2015;66:602-612.

24. Horowitz B, Miskulin D, Zager P. Epidemiology of hypertension in CKD. Adv Chronic Kidney Dis 2015;22:88-95.

25. Cohen JB, Cohen DL. Cardiovascular and renal effects of weight reduction in obesity and the metabolic syndrome. Curr Hypertens Rep 2015;17:34.

26. Athyros VG, Alexandrides TK, Bilianou H, et al. The use of statins alone, or in combination with pioglitazone and other drugs, for the treatment of non-alcoholic fatty liver disease/non-alcoholic steatohepatitis and related cardiovascular risk. An Expert Panel Statement. Metabolism 2017;71:17-32.

27. Aneni EC, Oni ET, Martin SS, et al. Blood pressure is associated with the presence and severity of nonalcoholic fatty liver disease across the spectrum of cardiometabolic risk. J Hypertens 2015;33:1207-1214.

28. Lonardo A, Ballestri S, Marchesini G, Angulo P, Loria P. Nonalcoholic fatty liver disease: a precursor of the metabolic syndrome. Dig Liver Dis 2015;47:181-190.

29. Ballestri S, Nascimbeni F, Romagnoli D, Lonardo A. The independent predictors of non-alcoholic steatohepatitis and its individual histological features: insulin resistance, serum uric acid, metabolic syndrome, alanine aminotransferase and serum total cholesterol are a clue to pathogenesis and candidate targets for treatment. Hepatol Res 2016;46:1074-1087.

30. Wree A, Broderick L, Canbay A, Hoffman HM, Feldstein AE. From NAFLD to NASH to cirrhosis-new insights into disease mechanisms. Nat Rev Gastroenterol Hepatol 2013;10:627-636.

31. Marchesini G, Petta S, Dalle Grave R. Diet, weight loss, and liver health in nonalcoholic fatty liver disease: Pathophysiology, evidence, and practice. Hepatology 2016;6:2032-2043.

32. Puppala J, Siddapuram SP, Akka J, Munshi A. Genetics of nonalcoholic fatty liver disease: an overview. $J$ Genet Genomics 2013;40:15-22.

33. Kawano Y, Cohen DE. Mechanisms of hepatic triglyceride accumulation in non-alcoholic fatty liver disease. J Gastroenterol 2013;48:434-441.

34. Pessayre D. Role of mitochondria in non-alcoholic fatty liver disease. J Gastroenterol Hepatol 2007;22 Suppl 1:S20-S27.

35. Rector RS, Thyfault JP, Uptergrove GM, et al. Mitochondrial dysfunction precedes insulin resistance and hepatic steatosis and contributes to the natural history of non-alcoholic fatty liver disease in an obese rodent model. J Hepatol 2010;52:727-736.

36. Amir M, Czaja MJ. Autophagy in nonalcoholic steatohepatitis. Expert Rev Gastroenterol Hepatol 2011;5:159-166.

37. Bechmann LP, Hannivoort RA, Gerken G, Hotamisligil GS, Trauner M, Canbay A. The interaction of hepatic lipid and glucose metabolism in liver diseases. J Hepatol 2012;56:952-964.

38. Vescovo T, Romagnoli A, Perdomo AB, et al. Autophagy protects cells from $\mathrm{HCV}$-induced defects in lipid metabolism. Gastroenterology 2012;142:644-653.

39. Nascimbeni F, Pais R, Bellentani S, et al. From NAFLD in clinical practice to answers from guidelines. J Hepatol 2013;59:859-871.

40. Hyötyläinen T, Jerby L, Petäjä EM, et al. Genome-scale study reveals reduced metabolic adaptability in patients with non-alcoholic fatty liver disease. Nat Commun 2016;7:8994.

41. Naik A, Košir R, Rozman D. Genomic aspects of NAFLD pathogenesis. Genomics 2013;102:84-95.

42. den Hoed M, Eijgelsheim M, Esko $T$, et al; CHARGE-AF Consortium. Identification of heart rate-associated loci and their effects on cardiac conduction and rhythm disorders. Nat Genet 2013;45:621-631.

43. Chatterjee A, Basu A, Chowdhury A, et al. Comparative analyses of genetic risk prediction methods reveal extreme diversity of genetic predisposition to nonalcoholic fatty liver disease (NAFLD) among ethnic populations of India. J Genet 2015;94:105-113.

44. Chen LZ, Xin YN, Geng N, Jiang M, Zhang DD, Xuan SY. PNPLA3 I148M variant in nonalcoholic fatty liver disease: demographic and ethnic characteristics and the role of the variant in nonalcoholic fatty liver fibrosis. World J Gastroenterol 2015;21:794-802.

45. Lim JW, Dillon J, Miller M. Proteomic and genomic studies of nonalcoholic fatty liver disease-clues in the pathogenesis. World $J$ Gastroenterol 2014;20:8325-8340.

46. Hoekstra M, Li Z, Kruijt JK, Van Eck M, Van Berkel TJ, Kuiper J. The expression level of non-alcoholic fatty liver disease-related gene PNPLA3 in hepatocytes is highly influenced by hepatic lipid status. J Hepatol 2010;52:244-251.

47. Kotronen A, Peltonen M, Hakkarainen A, et al. Prediction of non-alcoholic fatty liver disease and liver fat using metabolic and genetic factors. Gastroenterology 2009;137:865-872.

48. Pawlak M, Lefebvre P, Staels B. Molecular mechanism of PPARa action and its impact on lipid metabolism, inflammation and fibrosis in non-alcoholic fatty liver disease. J Hepatol 2015;62:720-733.

49. Francque S, Verrijken A, Caron S, et al. PPARa gene expression correlates with severity and histological treatment response in patients with non-alcoholic steatohepatitis. J Hepatol 2015;63:164-173.

50. Munroe PB, Barnes MR, Caulfield MJ. Advances in blood pressure genomics. Circ Res 2013;112:1365-1379.

51. Hotamisligil GS. Endoplasmic reticulum stress and the inflammatory basis of metabolic disease. Cell 2010;140:900-917.

52. Meli R, Mattace Raso G, Calignano A. Role of innate immune response in non-alcoholic Fatty liver disease: metabolic complications and therapeutic tools. Front Immunol 2014;5:177.

53. Stojsavljević S, Gomerčić Palčić M, Virović Jukić L, Smirčić Duvnjak L, Duvnjak M. Adipokines and proinflammatory cytokines, the key mediators in the pathogenesis of nonalcoholic 
fatty liver disease. World J Gastroenterol 2014;20:18070-18091.

54. Mian MO, Paradis P, Schiffrin EL. Innate immunity in hypertension. Curr Hypertens Rep 2014;16:413.

55. Johnson RJ, Lanaspa MA, Gabriela Sánchez-Lozada L, RodriguezIturbe $\mathrm{B}$. The discovery of hypertension: evolving views on the role of the kidneys, and current hot topics. Am J Physiol Renal Physiol 2015;308:F167-F178.

56. Mells JE, Anania FA. The role of gastrointestinal hormones in hepatic lipid metabolism. Semin Liver Dis 2013;33:343-357.

57. Johns EJ. The neural regulation of the kidney in hypertension and renal failure. Exp Physiol 2014;99:289-294.

58. Judd EK, Calhoun DA, Warnock DG. Pathophysiology and treatment of resistant hypertension: the role of aldosterone and amiloride-sensitive sodium channels. Semin Nephrol 2014;34:532-539.

59. Ilan Y. Leaky gut and the liver: a role for bacterial translocation in nonalcoholic steatohepatitis. World $J$ Gastroenterol 2012;18:2609-2618.

60. Zhu L, Baker RD, Baker SS. Gut microbiome and nonalcoholic fatty liver diseases. Pediatr Res 2015;77:245-251.

61. Jose PA, Raj D. Gut microbiota in hypertension. Curr Opin Nephrol Hypertens 2015;24:403-409.

62. Anstee QM, Day CP. The genetics of NAFLD. Nat Rev Gastroenterol Hepatol 2013;10:645-655.

63. Singh S, Allen AM, Wang Z, Prokop LJ, Murad MH, Loomba R. Fibrosis progression in nonalcoholic fatty liver vs nonalcoholic steatohepatitis: a systematic review and meta-analysis of pairedbiopsy studies. Clin Gastroenterol Hepatol 2015;13:643-654.e1-9; quiz e39-40.

64. Rinella ME, Sanyal AJ. Management of NAFLD: a stage-based approach. Nat Rev Gastroenterol Hepatol 2016;13:196-205.

65. Margini C, Dufour JF. The story of HCC in NAFLD: from epidemiology, across pathogenesis, to prevention and treatment. Liver Int 2016;36:317-324.

66. Piscaglia F, Svegliati-Baroni G, Barchetti A, et al; HCC-NAFLD Italian Study Group. Clinical patterns of hepatocellular carcinoma in nonalcoholic fatty liver disease: A multicenter prospective study. Hepatology 2016;63:827-838.

67. Fargion S, Porzio M, Fracanzani AL. Nonalcoholic fatty liver disease and vascular disease: state-of-the-art. World J Gastroenterol 2014;20:13306-13324.

68. Lonardo A, Sookoian S, Pirola CJ, Targher G. Non-alcoholic fatty liver disease and risk of cardiovascular disease. Metabolism 2016;65:1136-1150.

69. Athyros VG, Tziomalos K, Katsiki N, Doumas M, Karagiannis A, Mikhailidis DP. Cardiovascular risk across the histological spectrum and the clinical manifestations of non-alcoholic fatty liver disease: An update. World J Gastroenterol 2015;21:6820-6834.

70. Arima H, Barzi F, Chalmers J. Mortality patterns in hypertension. J Hypertens 2011;29 Suppl 1:S3-S7.

71. Neuman MG, Cohen LB, Nanau RM. Biomarkers in nonalcoholic fatty liver disease. Can J Gastroenterol Hepatol 2014;28:607-618.

72. 'O'Donnell MJ, Mente A, Smyth A, et. Salt intake and cardiovascular disease: why are the data inconsistent? Eur Heart $J$ 2013;34:1034-1040.

73. 'O'Donnell M, Mente A, Rangarajan S, et al; PURE Investigators. Urinary sodium and potassium excretion, mortality, and cardiovascular events. N Engl J Med 2014;371:612-623.

74. Younossi ZM, Reyes MJ, Mishra A, Mehta R, Henry L. Systematic review with meta-analysis: non-alcoholic steatohepatitis - a case for personalised treatment based on pathogenic targets. Aliment Pharmacol Ther 2014;39:3-14.

75. Pang RW, Poon RT. From molecular biology to targeted therapies for hepatocellular carcinoma: the future is now. Oncology 2007;72 Suppl 1:30-44.

76. Messerli FH, Makani H, Benjo A, Romero J, Alviar C, Bangalore
S. Antihypertensive efficacy of hydrochlorothiazide as evaluated by ambulatory blood pressure monitoring: a meta-analysis of randomized trials. J Am Coll Cardiol 2011;57:590-600.

77. James PA, Oparil S, Carter BL, et al. 2014 evidence-based guideline for the management of high blood pressure in adults: report from the panel members appointed to the Eighth Joint National Committee (JNC 8). JAMA 2014;311:507-520.

78. Gupta AK, Arshad S, Poulter NR. Compliance, safety, and effectiveness of fixed-dose combinations of antihypertensive agents: a meta-analysis. Hypertension 2010;55:399-407.

79. Zanchetti A, Thomopoulos C, Parati G. Randomized controlled trials of blood pressure lowering in hypertension: a critical reappraisal. Circ Res 2015;116:1058-1073.

80. McCormack T, Krause T, 'O'Flynn N. Management of hypertension in adults in primary care: NICE guideline. $\mathrm{Br} \mathrm{J}$ Gen Pract 2012;62:163-164.

81. Benlagha K, Weiss A, Beavis A, Teyton L, Bendelac A. In vivo identification of glycolipid antigen-specific T cells using fluorescent CD1d tetramers. J Exp Med 2000;191:1895-1903.

82. Boyault S, Rickman DS, de Reyniès A, et al. Transcriptome classification of HCC is related to gene alterations and to new therapeutic targets. Hepatology 2007;45:42-52.

83. McPherson S, Wilkinson N, Tiniakos D, et al. A randomised controlled trial of losartan as an anti-fibrotic agent in non-alcoholic steatohepatitis. PLoS One 2017;12:e0175717.

84. Tsuchiya S, Amano Y, Isono O, et al. Pharmacological evaluation of pioglitazone and candesartan cilexetil in a novel mouse model of non-alcoholic steatohepatitis, modified choline-deficient, amino acid-defined diet fed low-density lipoprotein receptor knockout mice. Hepatol Res 2017;47:584-592.

85. Namisaki T, Noguchi R, Moriya K, et al. Beneficial effects of combined ursodeoxycholic acid and angiotensin-II type 1 receptor blocker on hepatic fibrogenesis in a rat model of nonalcoholic steatohepatitis. J Gastroenterol 2016;51:162-172.

86. Georgescu EF, Ionescu R, Niculescu M, Mogoanta L, Vancica L. Angiotensin-receptor blockers as therapy for mild-to-moderate hypertension-associated non-alcoholic steatohepatitis. World $J$ Gastroenterol 2009;15:942-954.

87. Kudo H, Yata Y, Takahara $T$, et al. Telmisartan attenuates progression of steatohepatitis in mice: role of hepatic macrophage infiltration and effects on adipose tissue. Liver Int 2009;29:988-996.

88. Kato J, Koda M, Kishina M, et al. Therapeutic effects of angiotensin II type 1 receptor blocker, irbesartan, on non-alcoholic steatohepatitis using FLS-ob/ob male mice. Int J Mol Med 2012;30:107-113.

89. Rotman Y, Sanyal AJ. Current and upcoming pharmacotherapy for non-alcoholic fatty liver disease. Gut 2017;66:180-190.

90. Ilan Y. Review article: novel methods for the treatment of nonalcoholic steatohepatitis - targeting the gut immune system to decrease the systemic inflammatory response without immune suppression. Aliment Pharmacol Ther 2016;44:1168-1182.

91. Lalazar G, Mizrahi M, Turgeman I, et al. Oral administration of OKT3 MAb to patients with NASH, promotes regulatory T-cell induction, and alleviates insulin resistance: results of a phase IIa blinded placebo-controlled trial. J Clin Immunol 2015;35:399-407.

92. Chalasani N, Younossi Z, Lavine JE, et al; American College of Gastroenterology. The diagnosis and management of nonalcoholic fatty liver disease: practice guideline by the American Gastroenterological Association, American Association for the Study of Liver Diseases, and American College of Gastroenterology. Gastroenterology 2012;142:1592-1609.

93. Rubino F, Nathan DM, Eckel RH, et al; Delegates of the $2^{\text {nd }}$ Diabetes Surgery Summit. Metabolic surgery in the treatment algorithm for type 2 diabetes: a joint statement by International Diabetes Organizations. Diabetes Care 2016;39:861-877.

94. Sanyal AJ, Chalasani N, Kowdley KV, et al; NASH CRN. Pioglitazone, vitamin E, or placebo for nonalcoholic steatohepatitis. 
N Engl J Med 2010;362:1675-1685.

95. Neuschwander-Tetri BA, Loomba R, Sanyal AJ, et al; NASH Clinical Research Network. Farnesoid X nuclear receptor ligand obeticholic acid for non-cirrhotic, non-alcoholic steatohepatitis (FLINT): a multicentre, randomised, placebo-controlled trial. Lancet 2015;385:956-965.

96. Armstrong MJ, Gaunt P, Aithal GP, et al; LEAN trial team. Liraglutide safety and efficacy in patients with non-alcoholic steatohepatitis (LEAN): a multicentre, double-blind, randomised, placebo-controlled phase 2 study. Lancet 2016;387:679-690.

97. Ratziu V, Harrison SA, Francque S, et al; GOLDEN-505 Investigator Study Group. Elafibranor, an agonist of the peroxisome proliferatoractivated receptor- $\alpha$ and $-\delta$, induces resolution of nonalcoholic steatohepatitis without fibrosis worsening. Gastroenterology 2016;150:1147-1159.

98. Pearlman M, Loomba R. State of the art: treatment of nonalcoholic steatohepatitis. Curr Opin Gastroenterol 2014;30:223-237.

99. Yilmaz Y. Serum proteomics for biomarker discovery in nonalcoholic fatty liver disease. Clin Chim Acta 2012;413:11901193.

100.Li L, Lu DZ, Li YM, Zhang XQ, Zhou XX, Jin X. Proteomic analysis of liver mitochondria from rats with nonalcoholic steatohepatitis. World J Gastroenterol 2014;20:4778-4786.

101. Miller MH, Walsh SV, Atrih A, Huang JT, Ferguson MA, Dillon JF. Serum proteome of nonalcoholic fatty liver disease: a multimodal approach to discovery of biomarkers of nonalcoholic steatohepatitis. J Gastroenterol Hepatol 2014;29:1839-1847.

102.Dumas ME, Kinross J, Nicholson JK. Metabolic phenotyping and systems biology approaches to understanding metabolic syndrome and fatty liver disease. Gastroenterology 2014;146:46-62.

103.von Schönfels W, Patsenker E, Fahrner R, et al. Metabolomic tissue signature in human non-alcoholic fatty liver disease identifies protective candidate metabolites. Liver Int 2015;35:207-214.

104.Rodríguez-Gallego E, Guirro M, Riera-Borrull M, et al. Mapping of the circulating metabolome reveals $\alpha$-ketoglutarate as a predictor of morbid obesity-associated non-alcoholic fatty liver disease. Int $J$ Obes (Lond) 2015;39:279-287.

105. Ferslew BC, Xie G, Johnston CK, et al. Altered bile acid metabolome in patients with nonalcoholic steatohepatitis. Dig Dis Sci 2015;60:3318-3328.

106.Ilan Y. Compounds of the sphingomyelin-ceramide- glycosphingolipid pathways as secondary messenger molecules: new targets for novel therapies for fatty liver disease and insulin resistance. Am J Physiol Gastrointest Liver Physiol 2016;310:G1102-G1117.

107.Anjani K, Lhomme M, Sokolovska N, et al. Circulating phospholipid profiling identifies portal contribution to NASH signature in obesity. J Hepatol 2015;62:905-912.

108. Gorden DL, Ivanova PT, Myers DS, et al. Increased diacylglycerols characterize hepatic lipid changes in progression of human nonalcoholic fatty liver disease; comparison to a murine model. PLoS One 2011;6:e22775.

109.Luukkonen PK, Zhou Y, Sädevirta S, et al. Hepatic ceramides dissociate steatosis and insulin resistance in patients with nonalcoholic fatty liver disease. J Hepatol 2016;64:1167-1175.

110.Birerdinc A, Younossi Z. Can NASH lipidome provide insight into the pathogenesis of obesity-related non-alcoholic fatty liver disease? J Hepatol 2015;62:761-762.

111.Zhou Y, Llauradó G, Orešič M, Hyötyläinen T, Orho-Melander $\mathrm{M}$, Yki-Järvinen $\mathrm{H}$. Circulating triacylglycerol signatures and insulin sensitivity in NAFLD associated with the E167K variant in TM6SF2. J Hepatol 2015;62:657-663.

112. Muir K, Hazim A, He Y, et al. Proteomic and lipidomic signatures of lipid metabolism in NASH-associated hepatocellular carcinoma. Cancer Res 2013;73:4722-4731.

113. Morita Y, Sakaguchi T, Ikegami K, et al. Lysophosphatidylcholine acyltransferase 1 altered phospholipid composition and regulated hepatoma progression. J Hepatol 2013;59:292-299.

114.Henao-Mejia J, Elinav E, Jin C, et al. Inflammasome-mediated dysbiosis regulates progression of NAFLD and obesity. Nature 2012;482:179-185.

115. Yoshimoto S, Loo TM, Atarashi K, et al. Obesity-induced gut microbial metabolite promotes liver cancer through senescence secretome. Nature 2013;499:97-101.

116. Mehal WZ. The Gordian Knot of dysbiosis, obesity and NAFLD. Nat Rev Gastroenterol Hepatol 2013;10:637-644.

117.Usami M, Miyoshi M, Yamashita H. Gut microbiota and host metabolism in liver cirrhosis. World J Gastroenterol 2015;21:1159711608. 